\title{
On-Tissue Derivatization Strategy for Mass Spectrometry Imaging of Carboxyl-Containing Metabolites in Biological Tissues
}

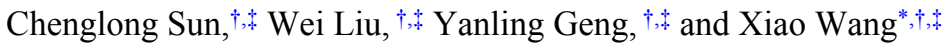 \\ $\dagger$ School of Pharmaceutical Sciences, Qilu University of Technology (Shandong Academy of Sciences), Jinan \\ 250014, China. \\ $\$$ Key Laboratory for Applied Technology of Sophisticated Analytical Instruments of Shandong Province, Shandong \\ Analysis and Test Center, Qilu University of Technology (Shandong Academy of Sciences), Jinan 250014, China.
}

This supporting information includes details on the chemicals and reagents, synthesis of TMPA, MALDI-MSI data processing, on-tissue MS/MS experiment. The synthesis of TMPA, the high-resolution MS spectrum and MS/MS spectrum of TMPA, the process to prepare spray models, the in situ MS/MS spectra of representative TMPA-CCM derivatives, the comparison between untreated and on-tissue derivatization strategy, were also investigated in the supporting information. Several supplementary figures and tables are provided as the references in the main text.

\section{Table of contents}

\begin{tabular}{lll}
\hline Figure & Title & Page \\
\hline Scheme S1 & Synthesis reaction of TMPA. & S3 \\
Figure S1 & The high-resolution MS spectrum and MS/MS spectrum of TMPA. & S3 \\
Figure S2 & The process to prepare spray models. & S4 \\
Figure S3 & $\begin{array}{l}\text { MS images and ion intensities of representative CCMs in untreated and } \\
\text { TMPA-derivatized spray models. }\end{array}$ & S4 \\
Figure S4 & $\begin{array}{l}\text { In situ MS/MS spectra of representative TMPA-CCM derivatives. (A) } \\
\text { TMPA-malic acid derivative; (B) TMPA derivative-stearic acid; (C) } \\
\text { TMPA-cholic acid derivative. }\end{array}$ & S5 \\
Figure S5 & $\begin{array}{l}\text { (A) Relative ion intensities of TMPA-CCM derivatives in rat kidney tissue } \\
\text { sections using on-tissue derivatization with different contents of TMPA. (B) MS } \\
\text { images and relative ion intensities of other metabolites in kidney tissue sections } \\
\text { with different contents of TMPA. ACA, arachidonic acid; CA, cholic acid; MAL, } \\
\text { malic acid. }\end{array}$ & S6 \\
\hline
\end{tabular}


Ion intensities of TMPA-CCM derivatives in rat kidney tissue sections at different reaction temperatures. ACA, arachidonic acid; CA, cholic acid; MAL, malic acid.

MS images of region-specific metabolites in untreated and derivatized brain sections.

Figure S7

MS images of region-specific metabolite ion in untreated and derivatized

Figure S8 esophageal sections.

MS images of TMPA-CCMs in kidney tissue sections. TCAs, tricarboxylic acid

Figure S9 cycle intermediates; FMA, fumaric acid; CTA, citric-acid; MLA, malic acid; SCA, succinic acid; ANA, aconitic acid; CA, cholic acid; GCA, glycocholic acid; CDCA, chenodeoxycholic acid.

Figure S10

MS images of representative metabolites in untreated and derivatized brain tissue sections.

Chemicals and Reagents. 1-(2-Dimethylaminoethyl)-piperazine (DMAP), iodomethane, O-(7-azabenzotriazol-1-yl)-N,N,N',N'-tetramethyluronium hexafluorophosphate (HATU), 1-hydroxybenzotriazole hydrate (HBOt), N-(3-dimethylaminopropyl)-N'-ethylcarbodiimide hydrochloride (EDC), triphenylphosphine (TPP), and 2,2'-dipyridyl disulfide (DPDS) were obtained from J\&K Chemical Ltd. (Beijing, China). Tricarboxylic acid cycle intermediate (TCA) standards (fumaric acid, malic acid, succinic acid, and citric acid), fatty acid standards (palmitic acid, stearic acid, oleic acid, linoleic acid, arachidonic acid, eicosapentaenoic acid, and docosahexaenoic acid), bile acid standards (cholic acid, chenodeoxycholic acid, and glycocholic acid), and 1,5-naphthalenediamine (1,5-DAN) were purchased from Aladdin Reagent Co. (Shanghai, China). Acetonitrile (ACN), acetone, diethyl ether, and N,N-dimethylformamide (DMF) were provided by Fuyu Fine Chemical Co., Ltd. (Tianjin, China).

Synthesis of TMPA. Briefly, the synthesis of TMPA was achieved by following steps: (i) 1-(2-Dimethylaminoethyl)-piperazine $(2.36 \mathrm{~g}, 15 \mathrm{mmol})$ dissolved in $100 \mathrm{~mL}$ diethyl ether; (ii) Iodomethane $(0.71 \mathrm{~g}$, $5 \mathrm{mmol}$ ) dissolved in $100 \mathrm{~mL}$ diethyl ether; (iii) mixing the solutions of 1-(2-Dimethylaminoethyl)-piperazine and iodomethane; (iv) After stirring at ambient temperature for $1 \mathrm{~h}$, the mixture was filtered and washed five times with diethyl ether and dried for further use. The synthesis reaction is shown in Scheme S1. The high-resolution MS and MS/MS spectrum of TMPA is shown in Figure S1.

MALDI-MSI data processing. The raw MALDI-MS data were imported into SCiLS Lab 2018b software to generate MS images. All the images were normalized to the total ion current. When imaging only one tissue section, relative intensities were adjusted on an individual basis to improve the quality of MS images. When comparing the MALDI-MSI data of different tissue sections, we can convert the individual sections into one data file through 
SCiLS Lab 2018b software. The newly generated file can directly characterize the difference of metabolites in different tissue sections.

On-tissue MS/MS experiment. The on-tissue MS/MS experiment was carried out on a rapiflex ${ }^{\mathrm{TM}}$ MALDI-TOF/TOF instrument (Bruker Daltonics, Billerica, MA). Laser repetition rate was operated at $5000 \mathrm{~Hz}$ and the MS/MS spectra were the sum of 20000 shots for precursor ions and product ions. The MS/MS spectra in the reflector mode were acquired with a sample rate reciprocal time of $0.4 \mathrm{~ns}$. The LasAtten value is $35 \%$. Ion source voltage 1 and Ion source voltage 2 were operated at $20 \mathrm{kV}$ and $19.45 \mathrm{kV}$, respectively. Reflector voltage 1, Reflector voltage 2, and Reflector voltage 3 were operated at $23.8 \mathrm{kV}, 1.79 \mathrm{kV}$, and $9.85 \mathrm{kV}$, respectively.

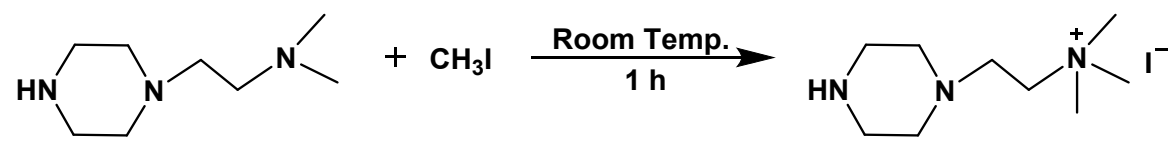

Scheme S1. Synthesis reaction of TMPA.
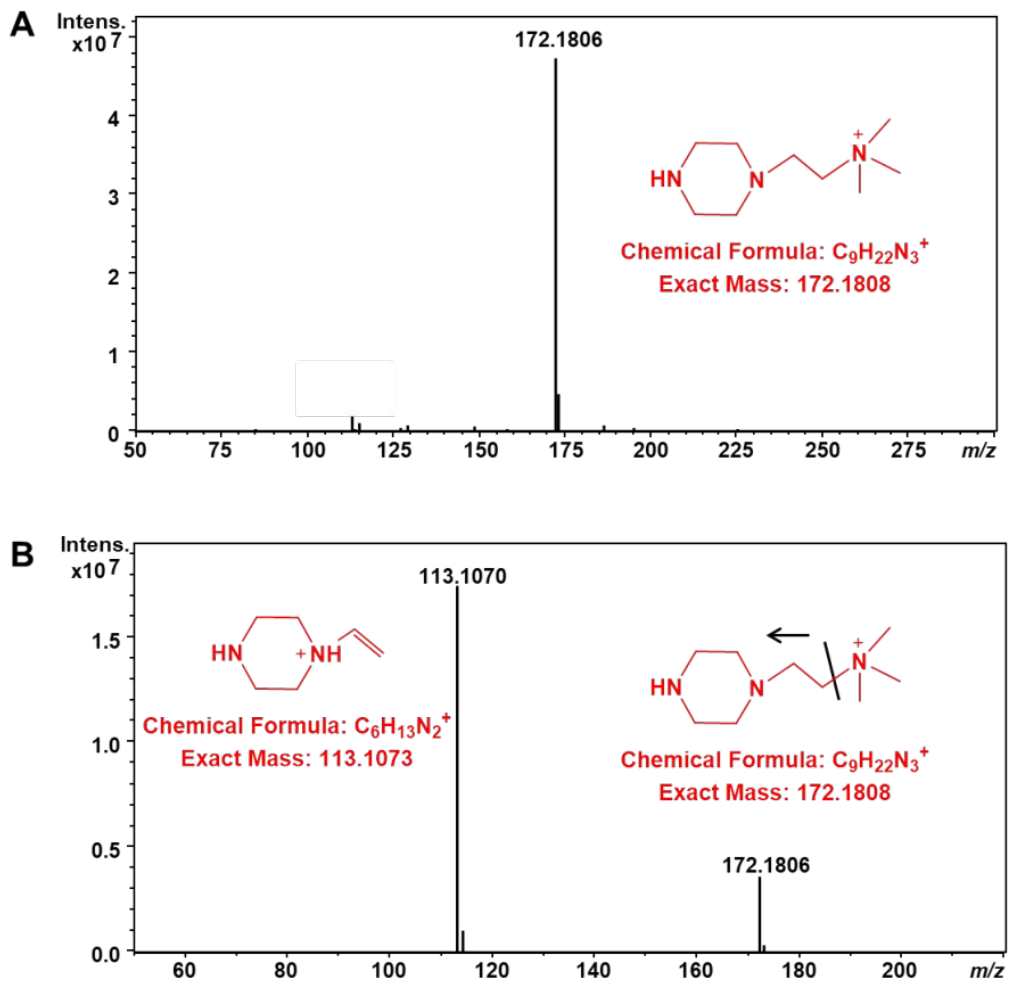

Figure S1. The high-resolution MS spectrum (A) and MS/MS spectrum (B) of TMPA. 


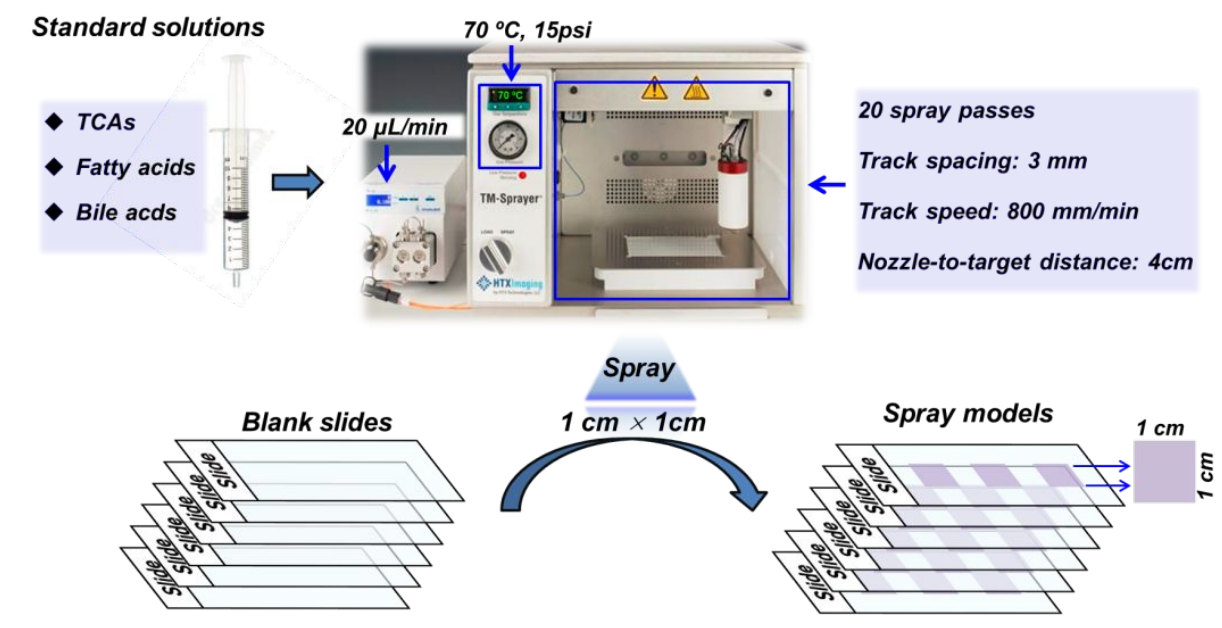

Figure S2. The process to prepare spray models.
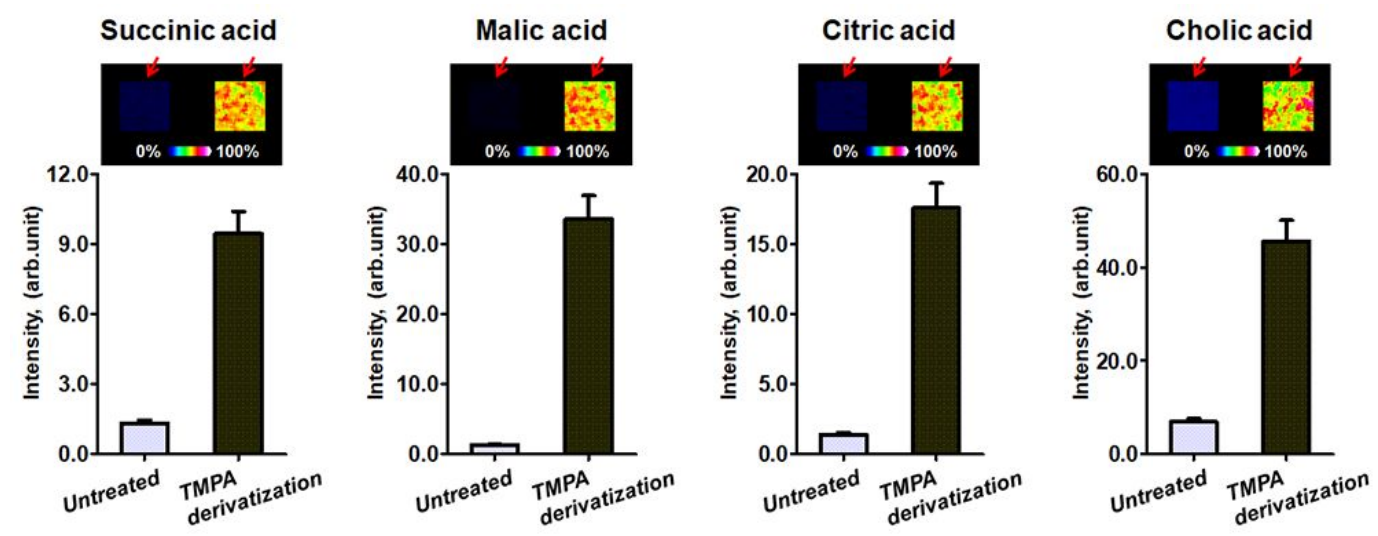

Figure S3. MS images and ion intensities of representative CCMs in untreated and TMPA-derivatized spray models. 

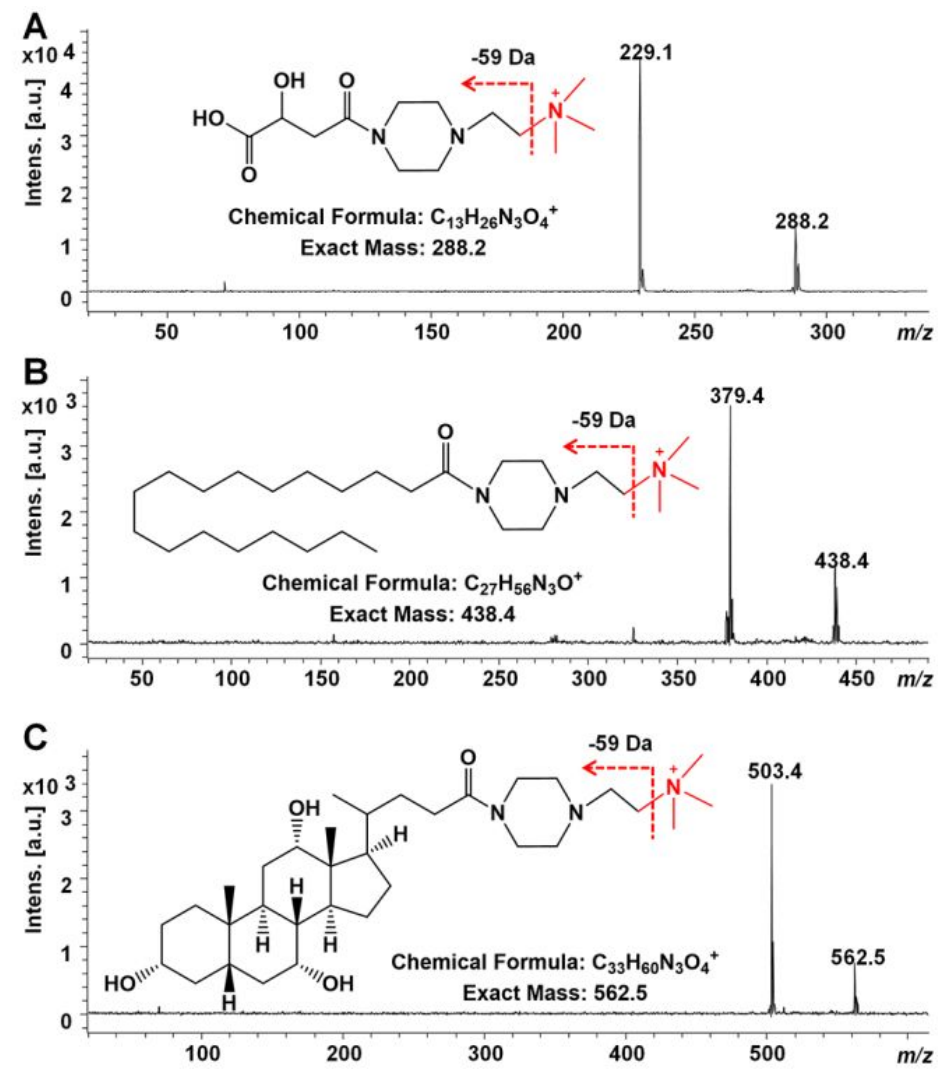

Figure S4. In situ MS/MS spectra of representative TMPA-CCM derivatives. (A) TMPA-malic acid derivative; (B) TMPA derivative-stearic acid; (C) TMPA-cholic acid derivative. 

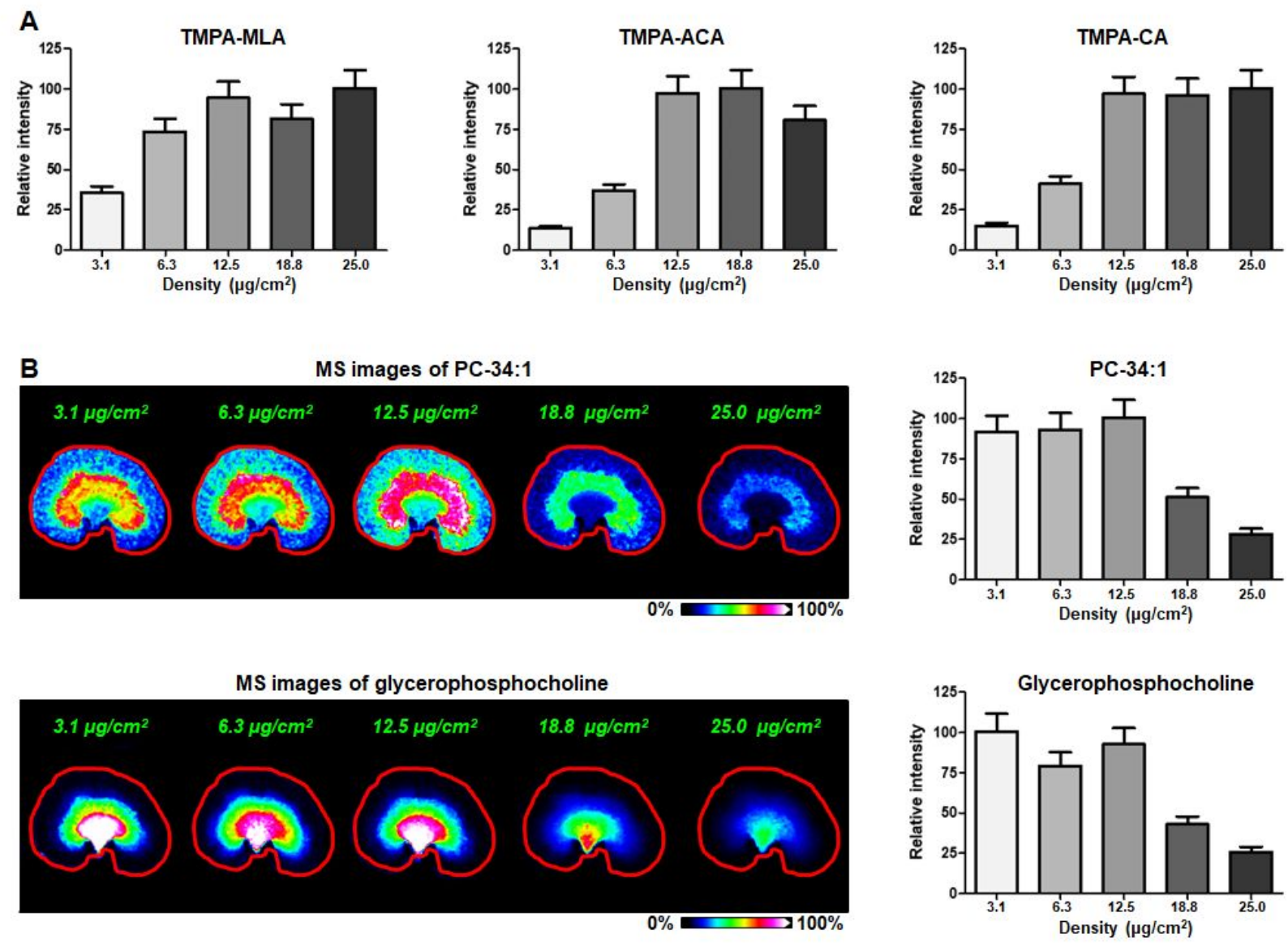

Figure S5. (A) Relative ion intensities of TMPA-CCM derivatives in rat kidney tissue sections using on-tissue derivatization with different contents of TMPA. (B) MS images and relative ion intensities of other metabolites in kidney tissue sections with different contents of TMPA. ACA, arachidonic acid; CA, cholic acid; MAL, malic acid.

TMPA-MLA

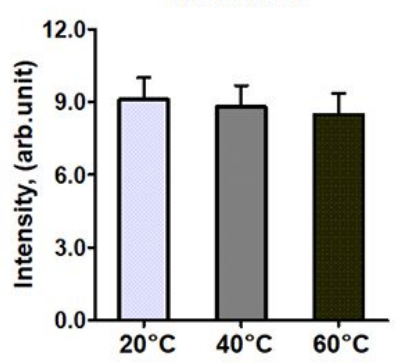

TMPA-ACA

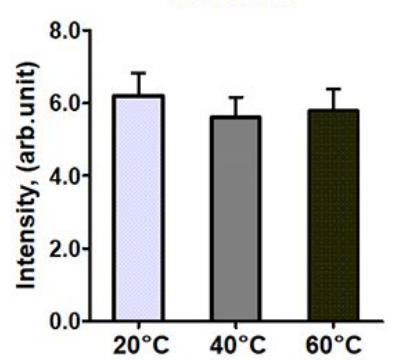

TMPA-CA

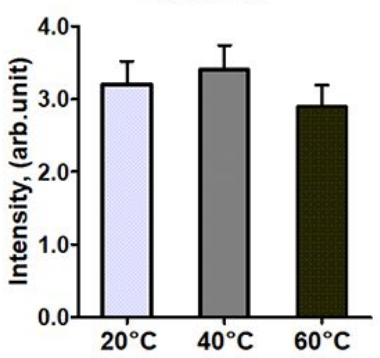

Figure S6. Ion intensities of TMPA-CCM derivatives in rat kidney tissue sections at different reaction temperatures. ACA, arachidonic acid; CA, cholic acid; MAL, malic acid. 

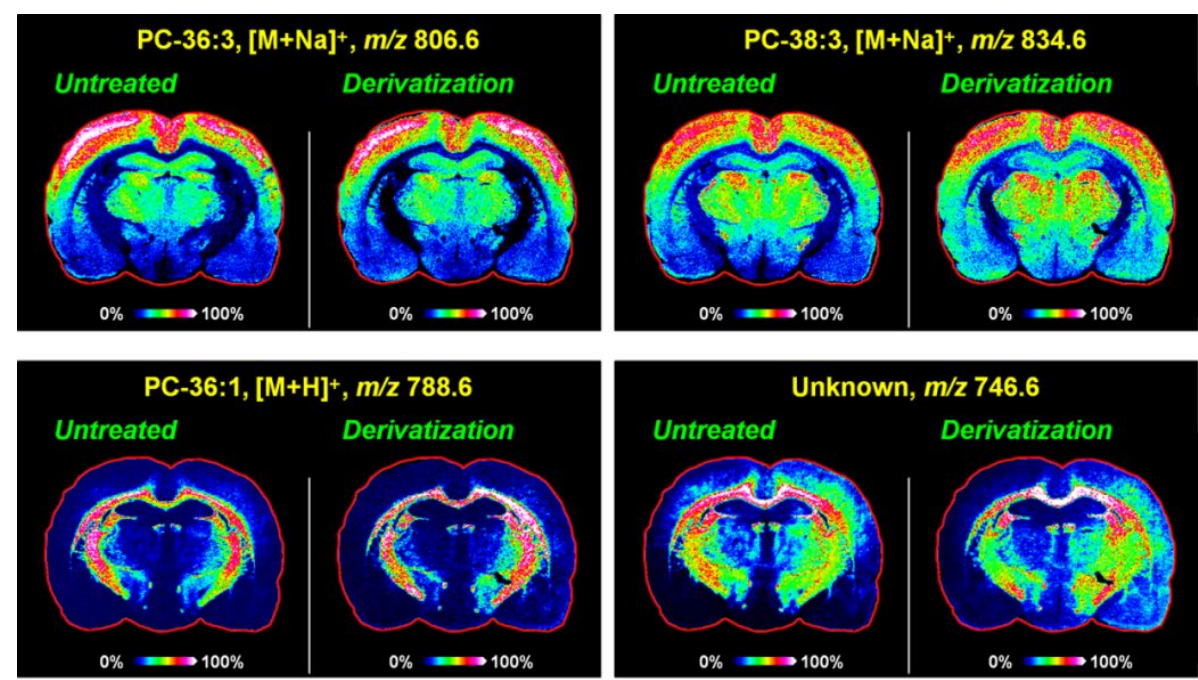

Figure S7. MS images of region-specific metabolites in untreated and derivatized brain sections.

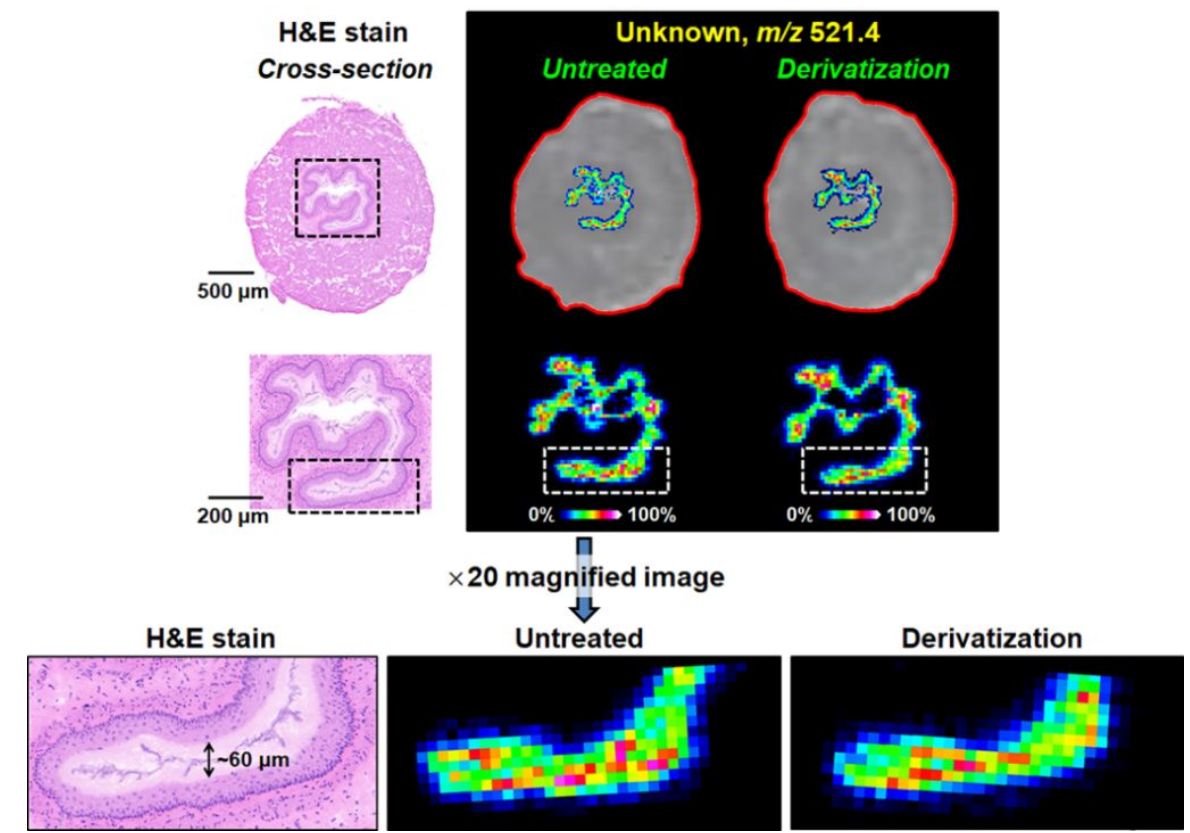

Figure S8. MS images of region-specific metabolite ion in untreated and derivatized esophageal sections. 


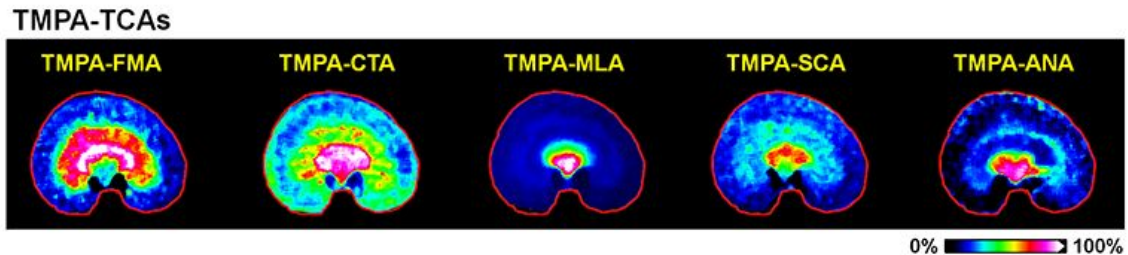

TMPA-fatty acids
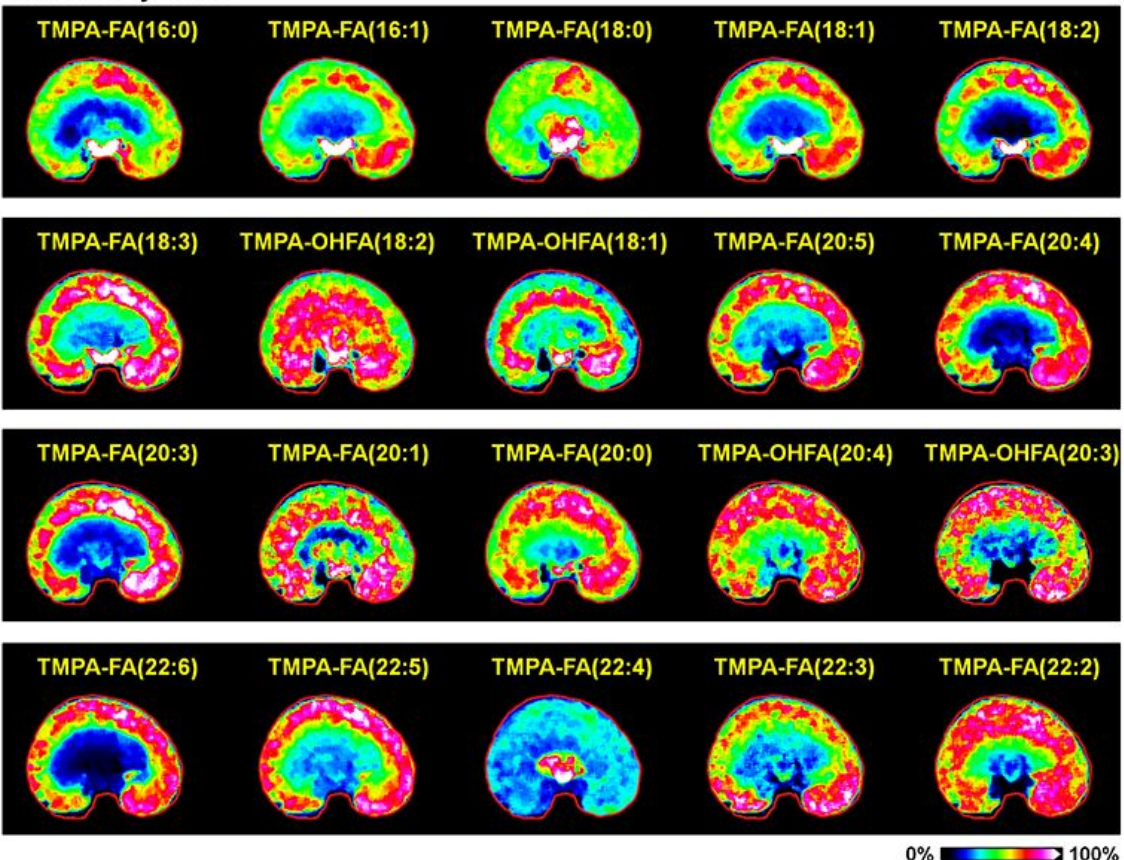

TMPA-bile acids

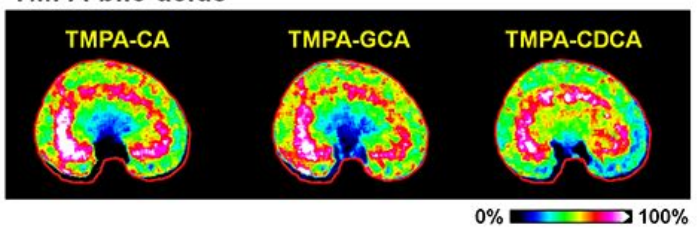

Figure S9. MS images of TMPA-CCMs in kidney tissue sections. TCAs, tricarboxylic acid cycle intermediates;

FMA, fumaric acid; CTA, citric-acid; MLA, malic acid; SCA, succinic acid; ANA, aconitic acid; CA, cholic acid; GCA, glycocholic acid; CDCA, chenodeoxycholic acid. 

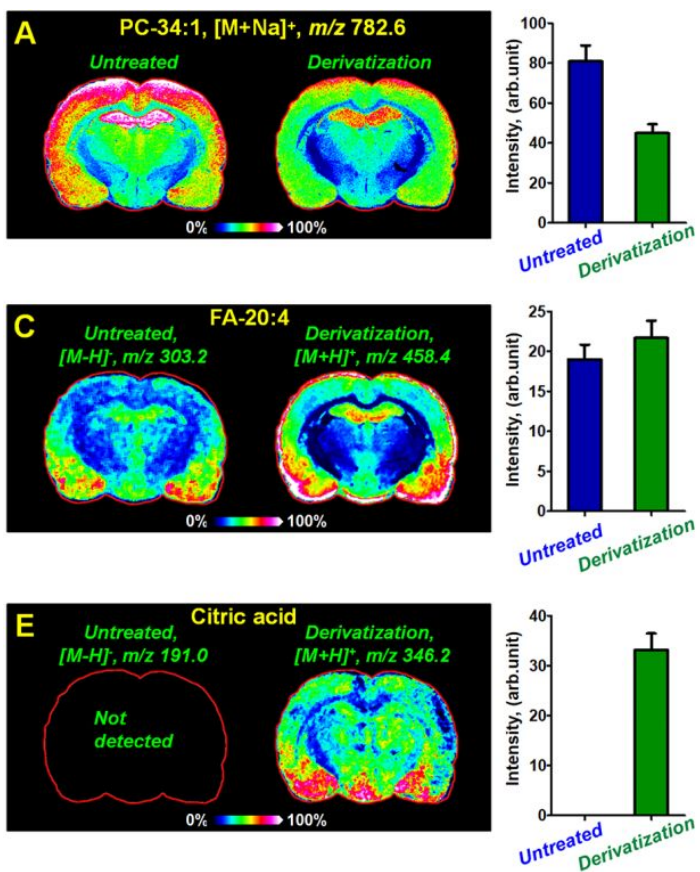
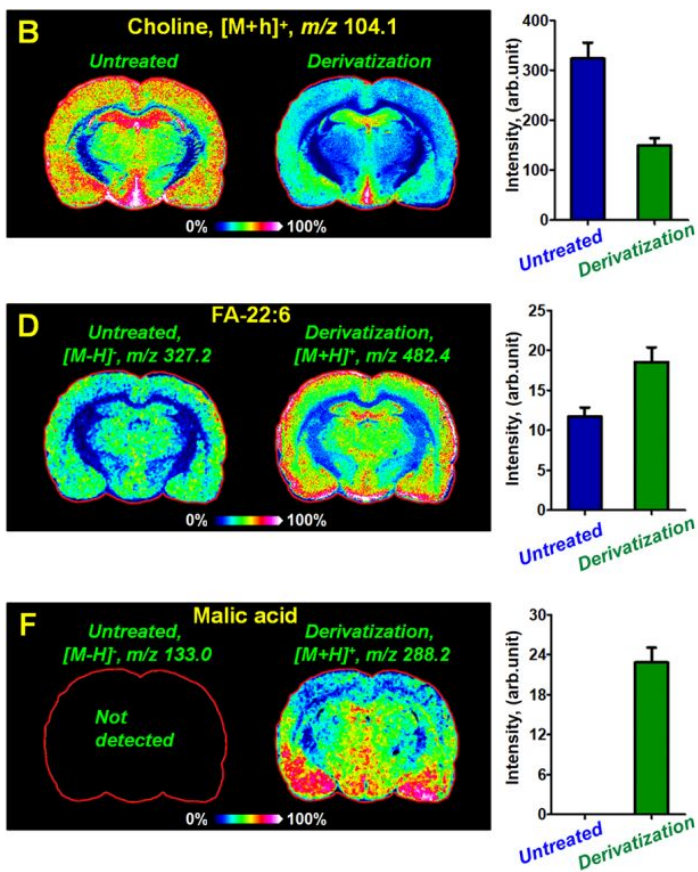

Figure S10. MS images of representative metabolites in untreated and derivatized brain tissue sections.

Table S1. Detail information of the TMPA derivatives of carboxyl-containing metabolites.

\begin{tabular}{|c|c|c|c|c|}
\hline Metabolites & $\begin{array}{c}\text { Molecular } \\
\text { formula }\end{array}$ & $\begin{array}{c}\text { Molecular } \\
\text { weight (Da) }\end{array}$ & $m / z$ of TMPA derivatives & $\begin{array}{c}\text { Product ions } \\
\text { of TMPA derivatives }\end{array}$ \\
\hline Fumaric acid & $\mathrm{C}_{4} \mathrm{H}_{3} \mathrm{O}_{4}$ & 116.0106 & 270.1808 & 211.1073 \\
\hline Succinic acid & $\mathrm{C}_{4} \mathrm{H}_{5} \mathrm{O}_{4}$ & 118.0262 & 272.1962 & 213.1229 \\
\hline Malic acid & $\mathrm{C}_{4} \mathrm{H}_{5} \mathrm{O}_{5}$ & 134.0212 & 288.1913 & 229.1178 \\
\hline Citric acid & $\mathrm{C}_{6} \mathrm{H}_{7} \mathrm{O}_{7}$ & 191.0267 & 346.1967 & 287.1232 \\
\hline Palmitic acid & $\mathrm{C}_{16} \mathrm{H}_{32} \mathrm{O}_{2}$ & 256.2402 & 410.4101 & 351.3365 \\
\hline Stearic acid & $\mathrm{C}_{18} \mathrm{H}_{36} \mathrm{O}_{2}$ & 284.2712 & 438.4414 & 379.3680 \\
\hline Oleic acid & $\mathrm{C}_{18} \mathrm{H}_{34} \mathrm{O}_{2}$ & 282.2555 & 436.4257 & 377.3522 \\
\hline Linoleic acid & $\mathrm{C}_{18} \mathrm{H}_{32} \mathrm{O}_{2}$ & 280.2401 & 434.4101 & 375.3365 \\
\hline Arachidonic acid & $\mathrm{C}_{20} \mathrm{H}_{32} \mathrm{O}_{2}$ & 304.2400 & 458.4100 & 399.3366 \\
\hline Eicosapentaenoic acid & $\mathrm{C}_{20} \mathrm{H}_{30} \mathrm{O}_{2}$ & 302.2241 & 456.3944 & 397.3210 \\
\hline Docosahexaenoic acid & $\mathrm{C}_{22} \mathrm{H}_{32} \mathrm{O}_{2}$ & 328.2400 & 482.4101 & 423.3366 \\
\hline Cholic acid & $\mathrm{C}_{24} \mathrm{H}_{40} \mathrm{O}_{5}$ & 408.2871 & 562.4573 & 503.3839 \\
\hline Chenodeoxycholic acid & $\mathrm{C}_{24} \mathrm{H}_{40} \mathrm{O}_{4}$ & 392.2923 & 546.4625 & 487.3890 \\
\hline Glycocholic acid & $\mathrm{C}_{26} \mathrm{H}_{43} \mathrm{NO}_{6}$ & 465.3087 & 619.4790 & 560.4053 \\
\hline
\end{tabular}

